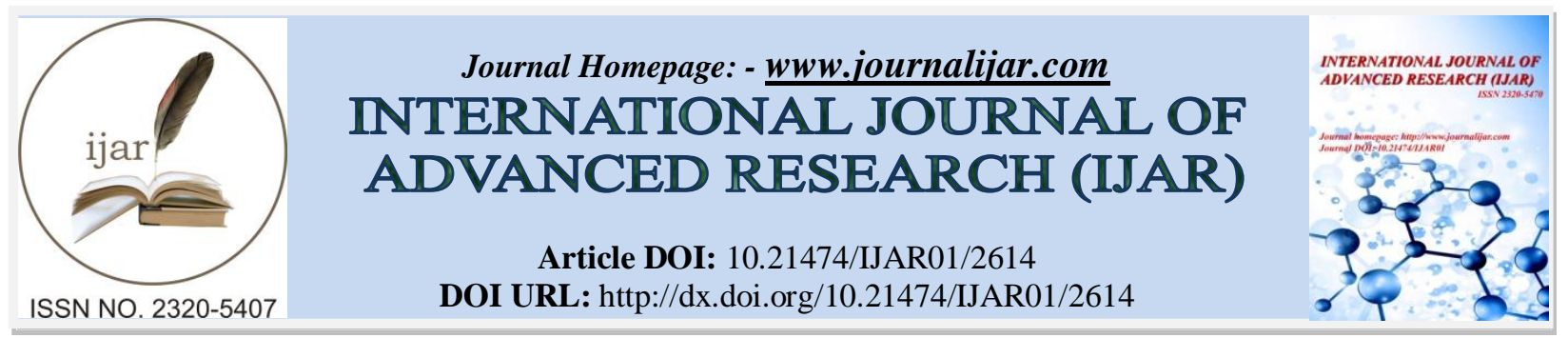

RESEARCH ARTICLE

\title{
CORRELATION BETWEEN THE INFORMANT QUESTIONNAIRE ON COGNITIVE DECLINE IN THE ELDERLY (IQCODE) AND MINI MENTAL STATE EXAMINATION (MMSE) AS A Screening TEST FOR AZHEIMER'S DISEASE.
}

Muhammad Kashif, Humaira Khalid, Fareed A Minhas and Manal Fatima.
Institute of Psychiatry \& WHO collaborating centre, Benazir Bhutto Hospital Rawalpindi-Pakistan.

\section{Manuscript Info}

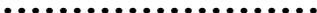

\section{Manuscript History}

Received: 30 October 2016

Final Accepted: 29 November 2016

Published: December 2016

Key words:-

Dementia, Cognitive impairment, MiniMental State Examination, Informant Questionnaire for Cognitive Decline in the Elderly.

\section{Abstract}

Objective: The objective of this study was to find the correlation and identification of the better of two commonly used Screening tools for detecting probable Cognitive impairment of the patients with Dementia.

Method: It was cross-sectional descriptive study. Non- probability convenient sampling technique was used to collect the data. The duration of the study was nine months i.e. May, 2008 to February, and 2009. Study Inclusion and exclusion criteria were observed. Patients were first screened on the scores of Mini Mental State Examination (MMSE) and then Informant Questionnaire on Cognitive Decline in Elderly (IQCODE).

Results: The findings of the study revealed that there was a negative correlation between MMSE Scores and IQCODE scores which was statistically significant.

Conclusion: To provide better screening, cognitive tests can be combined with informant- based tools. Overall, the results of our study are consistent with the findings of previous researches who reported that the IQCODE when filled out by an informant is a valid predictor of cognitive status and cognitive decline.

Copy Right, IJAR, 2016,. All rights reserved.

\section{Introduction:-}

It is observed that as the age progresses, there are some minor changes in memory which are considered as a normal part of the ageing. However, when these minor memory changes start to impact on normal day to day functioning, It is required to find out the root cause of this memory loss by detailed assessment so that which can be due to any health problem such as dementia. A detailed assessment or screening is required to be sure of cause of these memory changes. Cognitive impairment is a central and generally considered as a first indicator of Dementia (APA, 1994). To early diagnose Dementia efficiently quick and significant cognitive tests are required. According to The International Psychogeriatric Association survey, 20 brief cognitive scales which are used by clinicians in their practice due to their effectiveness, familiarity and ease in administration (Shulman et al., 2006). The most widely used tests are Mini-Mental State Examination (MMSE) and Informant Questionnaire for Cognitive Decline in the elderly (IQCODE).

The Mini Mental State Examination (MMSE) is most commonly used instrument by clinicians in their clinical practice \& research. . However, informant-based screening tests provide a complementary approach. They are 
effective for individuals with low levels of education and literacy, lack of co-operation or death and who are unable to undergo direct cognitive testing because of acute illness. To provide better screening, cognitive tests can be combined with informant- based tools. A wide range of informant- based tools are available which are not widely used ( Jorm, 1996). ${ }^{12}$

Different cultural and educational background accounts for different suitable instruments which would vary for each country. The most widely used tool for screening of Dementia is MMSE. It is easy \& quick to administered. The only drawback of MMSE is that it cannot be used with illiterate and hearing/visual deficits patients. Another tool for assessing cognitive deficits is IQ Code, which is used to access cognitive impairments by relying on informant's information related to the patient. This tool is used in all patients regardless of their education level or physical condition. ${ }^{9}$

A study conducted by O'Connor et al (1988) revealed that general practitioners know only $30 \%$ of mild suffers of Dementia and around $80 \%$ of patients with severe dementia ${ }^{36}$. A latest study findings revealed that in primary care systems, $2 / 3$ of the patients suffering from Dementia go undiagnosed. These facts does not comes as surprise because of the deterministic/ fatalistic attitude of clinicians regarding Dementia in general practice. One more study concluded that General practitioners did not give importance to diagnose Dementia at earliest because they feel it isn't significant to look for incurable disease. Usage of screening tools such as MMSE regularly in the primary care after age 75 can improve these undiagnosed or unrecognized Dementia.

A study done by Zanetti et al. ${ }^{16}$ found out that there is a discrepancy between informant's information and direct assessment of everyday activities of patients suffering from Mild or very mild Dementia due to informant's personal attributes. IQCODE is used as a screening tool for diagnosing Dementia if it reveals positive scores, this accounts for detailed clinical assessment to confirm the diagnosis. MMSE is a patient administered test which is easy and quick to use. This is helpful for both the clinician and the patient, informant is not required to complete the assessment. This is the biggest advantage of MMSE with one small drawback that it cannot be used with illetrate and visual/ hearing impaired patients. Worldwide in primary care settings, MMSE is used to screen patients for Dementia. ${ }^{17}$

A study done by Jorm and colleagues revealed that IQCODE score significantly correlates with MMSE scores, tests of mental processing speed and tests of episodic memory. Another study also showed correlation between MMSE and IQCODE scores. A recent study conducted by Isella and colleagues revealed that IQCODE plays a vital role in diagnosing and evaluating prognosis of patients suffering with Mild Cognitive Impairment (MCI). Lastly, researchers found out that using IQCODE with other neurological tests accounts for more dependable diagnosis of mild cognitive impairment (MCI) and Dementia.

Study Objective: The objective of this study was to identify the better of two commonly used Screening tools for detecting probable Cognitive impairment of the patients with Dementia. This is a co relational study of the Mini Mental State Examination and Informant Questionnaire on Cognitive Decline in the Elderly (IQCODE).

The aim of this study was to compare the Mini-Mental State Examination (MMSE) with the Informant Questionnaire for Cognitive Decline in the Elderly (IQCODE) in patients with Alzheimer Disease

\section{Method:-}

\section{Sample:-}

We have selected 45 participants of both genders. It is a cross-sectional descriptive research. Non- probability convenient sampling was done and inclusion criteria were (a) Patients above age of 65 years, (b) Fulfilling ICD-10 criteria for Alzheimer's disease (c) must accompanied by an attendant. Exclusion criteria were (a) Patients having serious co-morbid medical problems; uremia, hepatic encephalopathy, congestive cardiac failure, anemia, chronic pulmonary disease, hypertensive encephalopathy etc.

\section{Instruments:-}

\section{Mini Mental State Examination (MMSE):-}

The mini-mental state examination (MMSE) was developed by Folstein et al in 1975. The MMSE is quick and easy to administered depending on severity of impairment of person. There are 30 items which are divided into 5 main categories that are orientation, registration, attention and calculation, recall and language. There are 2 sections of 
this instrument. The first section comprises of verbal responses of individuals to orientation, attention and memory questions. The 2nd section consists of naming reading and writing and the ability to follow verbal and written commands, write a sentence and copy 5 a polygon (Folstein, Folstein \& McHugh.,1975). Due to copyright issues, the William Molloy version of MMSE is not available. This version is used to screen cognitive impairment, access cognitive changes and severity of cognitive impairment. MMSE has been translated into different languages and used as a cultural free instrument for screening cognitive decline. There is a modified version available for persons with hearing impairment. Total scores on MMSE is 30 points. Higher scores on each domain indicate higher functioning. There has been numerous debates over scoring and cut off points. Score less than 24 is used as a most widely cutoff points (Folstein et al 2001). Different populations, age and educational backgrounds accounts for diverse cutoff scores which is indicated by numerous studies levels (Lopez, Charter, Mostafavi, Nibut, \& Smith, 2005). Any motor/physical deficit can hinder the interpretation of scale if it is not identified in the initial stage for example a person who has hearing or motor impairment can't understand the instrument properly or maybe unable to perform in writing or drawing test.

Informant Questionnaire on Cognitive Decline in the Elderly (IQCODE):-

The Informant Questionnaire on Cognitive Decline in the Elderly (IQCODE) is a widely used instrument for screening of Dementia in elderly. It relies on informant's information about day to day cognitive functioning of patients with Dementia. The instrument is a retrospective questionnaire for the care givers to tell about the changes in patient's functional and cognitive skills in the past 10 years of his life. One advantage of using this instrument is that it can be used in any population irrespective of their education or literacy level.

ICODE is a 26 item informant rated questionnaire with five points likert scale ranging from 1-5. It is available in different versions such as face to face interview, self ratings, telephone and online versions. Total score is obtained by adding all the score of each item and dividing it by 26 .

The most favorable cut off score on the modified ICODE is 3.42, with the specificity and sensitivity of $95 \%$ and 90 $\%$ respectively. One advantage of using ICODE questionnaire is that it can be used in any population of irrespective of the educational level, premorbid ability in cultural dominant's language. The drawback of IQCODE is that it gets affected by caregivers characteristic such as Anxiety or Depression, and the type of relationship between the caregiver/ informant and the patient.

\section{Procedure:-}

Firstly, the procedure was explained to the patients and his/her attendants. Inform consent was taken and demographic form was filled for each patient then physical examination was done. Participants were diagnosed to have Alzheimer Disease on the basis of ICD-10 criteria. Potential participants were screened by administering Mini Mental State Examination (MMSE) those scoring 25/30 or below on MMSE then the informants were asked to think about the patient when he had not developed the illness and fill the questionnaire. Then they were asked to think of the way the patient was at the time of examination and fill the questionnaire again. Then, Informant Questionnaire on Cognitive Decline in the Elderly (IQ CODE) was applied.

\section{Result:-}

The study was conducted in the Institute of Psychiatry, Rawalpindi General Hospital, Rawalpindi, from May 01, 2006 to January 31, 2007.

A total of 60 patients fulfilling the study criteria were invited to participate in the study, 08 refused to participate while other 7 were excluded because they did not fulfill the inclusion criteria, 45 consented to participate and successfully completed the study protocol. Amongst the total 45 study participants 15 (33.3\%) were males and 30 $(66.6 \%)$ were females. The mean age of all the study participants was 74.80 years (S.D. +7.22 years), however the mean age of the male patients was 72.93 years (S.D. + 6.09 years), and mean age of female patients was 75.73 years (S.D. + 7.65 years). The marital status of the study participants revealed that $14(31.3 \%)$ were married and 31 $(68.9 \%)$ were widowed.

Iqcode:-

Cut off score of IQCODE was 3.3. 
Table 1 Results of IQCODE

\begin{tabular}{|c|c|c|}
\hline IQCODE Score & No of Patients & Percentage \\
\hline$<3.29$ & 1 & $2.2 \%$ \\
\hline $3.3-4.0$ & 5 & $11.0 \%$ \\
\hline 4.0 or $<$ & 39 & $86.8 \%$ \\
\hline Total & 45 & $100 \%$ \\
\hline
\end{tabular}

Table 1 shows the frequency distribution of IQCODE scores and their percentages. $13.2 \%$ of the informants had scores below 4.0. $86.8 \%$ of the sample had score 4 or greater than 4 . The cut off score of IQCODE is 3.3 .

Table 2:- Mean Differences and Standard Deviation of IQCODE scores and MMSE scores

\begin{tabular}{|c|c|c|c|}
\hline & Mean & Std. Deviation & $\mathrm{N}$ \\
\hline IQCODE & 4.5340 & .43063 & 45 \\
\hline MMSE & 14.60 & 5.096 & 45 \\
\hline
\end{tabular}

Table 2 shows mean differences and standard deviations of IQCODE and MMSE. Mean of IQCODE and MMSE was 4.53 and 14.60 respectively. Standard deviation of IQCODE was .43 and MMSE was 5.09.

Table 3:- Correlation Between IQCODE scores and MMSE score.

\begin{tabular}{|l|c|c|c|}
\hline \multirow{4}{*}{ IQCODE } & Pearson Correlation & IQCODE & MMSE \\
\cline { 2 - 4 } & Sig. (2-tailed) & 1 & $-.570(* *)$ \\
\cline { 2 - 4 } & $\mathrm{N}$ & 45 & .000 \\
\hline \multirow{4}{*}{ MMSE } & Pearson Correlation & $-.570(* *)$ & 45 \\
\cline { 2 - 4 } & Sig. (2-tailed) & .000 & 1 \\
\cline { 2 - 4 } & $\mathrm{N}$ & 45 & 45 \\
\hline
\end{tabular}

** Correlation is significant at the 0.01 level (2-tailed).

Table 3 shows correlation between IQCODE scores and MMSE scores. There was a negative correlation between MMSE Scores and IQCODE scores which was statistically significant (Pearson Correlation coefficient was -0.570 , $\alpha=0.01$ and $P$ value $=0.00)$. 
Table 5:- Scatter Plot between MMSE Scores and IQCODE Scores

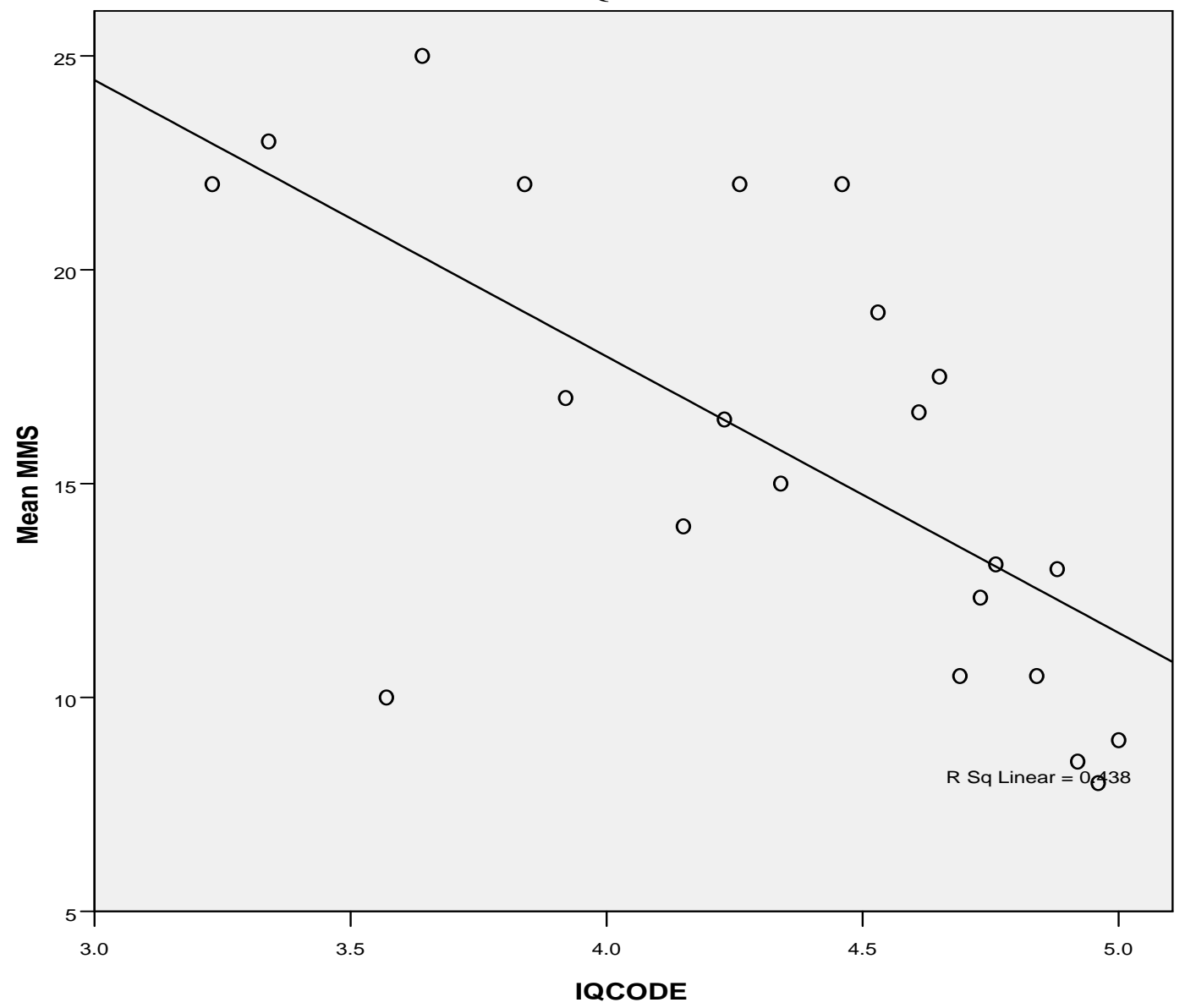

Above scatter plot showing negative correlation between IQCODE scores and MMSE scores.

\section{Discussion:-}

The purpose of this study was to determine the correlation between the mini mental state examination (MMSE) and Informant Questionnaire on Cognitive Decline (IQCODE). The results of this study revealed there was a significant negative correlation between the MMSE scores and IQCODE scores.

A study was done by Jorm et al 1991 in which they used combination of MMSE and ICODE test values. They divided MMSE and ICODE scores into deciles and added them. Nevertheless, combine scores were only slightly better at screening of dementia then the IQ Code.

Combination of test values has also been evaluated by Jorm et al. (1991). They divided IQCODE and MMSE scores into deciles and summed them. However, the combined scores were only marginally better at detecting dementia than the IQCODE alone. The IQCODE was found to perform at least as well as the MMSE against all diagnoses and significantly better when judged against the algorithmic ICD-10 diagnoses. Different studies have indicated that IQCODE is not influenced by the age and education of informant (Jorm et al., 1996), or by the type or length of relationship (Fuh et al., 1995).

A study done by Hancock and Larner (2009) revealed that combining the ICODE scores with MMSE or ACE-R greatly improved the specificity, accuracy and positive predictive values when they are used together but not when they are used in parallel.

Flicker et al. (1997) have also examined the "And" and "Or" rules by combining the IQCODE with either the MMSE or the Abbreviated Mental Test Score. Using the "OR" rule enhanced the sensitivity considerably, but at some expense to specificity, whereas using the "And" rule enhanced specificity, but at the expense of sensitivity. 
A Meta analysis was done to compare the ICODE and MMSE instruments which revealed that both the instruments have same effectiveness in screening of patients with Dementia. Nevertheless both the instruments are valid and sensitive in screening of Dementia. There are some differences between both the instruments but they share a similar sensitivity and specificity. MMSE is a patient administered instrument for cognitive evaluation whereas ICODE is a questionnaire based on informant's information about patient's cognitive functions for the past 10 years. However, MMSE is considered as a sensitive instrument regarding educational level whereas ICODE is not affected by education level. Regardless of the fact both the instruments are rated as good screening instrument for detecting Dementia. Hence, it is evident that the MMSE and the IQCODE identify different patients with different patterns of decline, but we have not found any studies that analyze these differences in depth.

Overall, the results of our study are consistent with the findings of Jorm and others who reported that the IQCODE when filled out by an informant is a valid predictor of cognitive status and cognitive decline.

\section{Limitations:-}

Limitations of this study are as follow:

- There was no control group to compare the results.

- Patients included in the study were referred by General Practitioners or were recruited from OPD so the sample might not be the representative of the target population.

- Because the sampling was not randomized, so it cannot be generalized to general population. .

- As this was a cross-sectional descriptive study, so we cannot comment on both the evolution and the course of Alzheimer's disease.

\section{Acknowledgment:-}

We would like to acknowledge all of those beings who helped us to complete this research work and to present the findings in a form of research article, sincere gratitude is hereby extended to those who never ceased their help till the completion of this research paper, thanks to Ms Faiza Aslam, Zubia Munawar and Mr Abdul Basit for their kind support and encouragement, we acknowledge their assistance. We tried our level best to ensure the accuracy of the material, to present the information through this paper as clearly as possible and to ensure that it would be comprehendible to the widest spectrum of researchers, analysts and students who want to pursue research on this domain later on. Last but not the least, we pay our deepest appreciation to all the patients and their attendants, without them this work was not possible.

\section{Conclusion:-}

This study shows that there is a negative correlation between MMSE Scores and IQCODE scores which was statistically significant. To provide better screening, cognitive tests can be combined with informant- based tools. There are bulk of previous studies that support our research findings, in one of the study findings support that combined scores were marginally better at detecting dementia than the IQCODE alone and was found to perform at least as well as MMSE against all diagnosis and significantly better judged against the algorithmic ICD-10 diagnosis. Overall, the results of our study are consistent with the findings of previous researches who reported that the IQCODE when filled out by an informant is a valid predictor of cognitive status and cognitive decline. 